Original Research Paper

\title{
Nondestructive Assessment of Egg Freshness using a Synchronous Fluorescence Spectral Technique
}

\author{
${ }^{1}$ Jianhu Wu, ${ }^{1}$ Guifeng Li, ${ }^{1,2 *}$ Yankun Peng, ${ }^{1}$ Junjie Du, ${ }^{1}$ Jianguo Xu and ${ }^{1}$ Gang Gao \\ ${ }^{1}$ College of Food Science, Shanxi Normal University, Lin fen, 041000 China \\ ${ }^{2}$ College of Engineering China Agricultural University, Beijing 100083, China
}

\author{
Article history \\ Received: 12-09-2019 \\ Revised: 09-11-2019 \\ Accepted: 14-12-2019 \\ Corresponding Author: \\ Yankun Peng \\ College of Food Science, \\ Shanxi Normal University, Lin \\ fen, 041000 China and \\ College of Engineering China \\ Agricultural University, \\ Beijing 100083, China \\ Email: ypeng@cau.edu.cn
}

\begin{abstract}
Freshness is an important index of egg quality. In this study, a synchronous fluorescence spectral technique was employed to determine the freshness of an intact egg. Synchronous fluorescence spectra of intact eggs were acquired using a fluorescence spectrometer supported by a laboratory fluorescence acquisition device and egg freshness (Haugh Unit) was obtained using destructive methods. Eggs feature fluorescence signals were mainly concentrated in two regions: A (excitation wavelength of 290 $\mathrm{nm}$ over the emission wavelength range of $320-380 \mathrm{~nm}$ ) and B (excitation wavelength range of $380-570 \mathrm{~nm}$ over the emission wavelength range of 610-735 nm). The two regions were selected as regions of interest, which include 2581 Excitation-Emission (Ex-Em) wavelengths; stepwise discrimination analysis was performed on the 2581 Ex-Em wavelengths to choose optimal Ex-Em wavelength combinations. A Multiple Linear Regression (MLR) prediction model was built using fluorescence signals based on the optimal Ex-Em wavelength combinations. The results revealed that the freshness of an egg could be accurately predicted with Rp2 of 0.8879 and a root mean square error estimated by validation (SEP) of 6.2896. This work demonstrates that the synchronous fluorescence spectral technique has high potential for nondestructive sensing of egg freshness.
\end{abstract}

Keywords: Synchronous Fluorescence Spectra, Eggs Freshness, Stepwise Discrimination Analysis, Multiple Linear Regressions

\section{Introduction}

Eggs contain many kinds of nutrients that the human body needs and are an important food for human beings. Freshness is an important index of egg quality; the freshness of eggs not only affects the taste of eggs and their products but also directly determines the absorption of protein and other nutrients. The Haugh unit is an important parameter for characterizing the freshness of eggs $(\mathrm{Li}, 2013)$ and is widely used for measuring egg freshness. however, it is a destructive method and cannot meet the requirements of modern rapid detection. In recent years, Near-Infrared (NIR) spectroscopy has been efficaciously used to quantify egg composition and as a tool for assessing freshness (Bamelis et al., 2003; Kemps et al., 2006; Zhao et al., 2010).

Fluorescence spectroscopy, which depends on sensing the contents of naturally occurring fluorescent compounds, has shown potential for rapid analysis of the quality of food products (Schneider et al., 2005;
Aït-Kaddou et al., 2011). In traditional fluorescence spectroscopy, a specific excitation wavelength of light irradiates the surface of a sample and the fluorophore of the specific excitation wavelength absorbs and emits energy in a fluorescent form. The amount of emitted energy and the wavelength at which the energy is emitted mainly depend on the fluorophore itself. Most foods, especially animal foods, contain a large number of quality-related fluorescent groups. Fluorescence spectra can provide information about the fluorescent groups in foods, such as proteins, amino acids, heterocyclic aromatic amines and so on (Aït-Kaddou et al., 2011; Brøndum et al., 2000). Therefore, fluorescence spectra technology can be used in food quality and safety study. and it has been applied in cereal, oil (Li et al., 2015), fruit and vegetable, honey (Lenhardt et al., 2015) for quality analysis, variety identification and adulteration detection. In recent years, traditional fluorescence technology has been widely used in meat quality and safety testing. The classification, grading, chemical composition and rheological parameters 
of beef tenderness (Bjørg et al., 2002; Sahar et al., 2009; Asylbek et al., 2012; Sahar and Dufour, 2015), lipid oxidation (Gatellier et al., 2009; Pouzo et al., 2016), microbial contamination and spoilage (Durek et al., 2016), reproductive age and texture characteristics (Kulmyrzaev et al., 2012) have been studied by conventional fluorescence detection. In addition, the quality evaluation of pork, such as the water holding capacity (Li et al., 2015), freshness indicators, such as the protoporphyrin content (Durek et al., 2012), ATP, contents of its degradation products and surface bacteria number (Oto et al., 2013), have also been studied. A few studies have also reported freshness analyses for fish (Dufour et al., 2003) and lipid oxidation (Gatellier et al., 2007) of chicken. While, traditional fluorescence spectroscopy relies on a few wavelengths of light to excite fluorescence spectra of a sample. A few excitation wavelengths are not enough to fully excite the fluorescence information of a sample (Mala et al., 2016).

Compared with traditional fluorescence spectroscopy, Synchronous Fluorescence Spectroscopy (SFS) could obtain spectral information for excitation and emission by successively projecting a continuous excitation light wave onto a sample over a certain range of wavelengths, which can be used to obtain fluorescence information for various fluorescent groups at the same time, has great potential to be used in the detection of complex samples such as meat and eggs. SFS technology has been applied in meat quality analyses, such as total bacterial count, fatty acid composition (Aït-Kaddour et al., 2016), discriminating between different cooking conditions (Sahar et al., 2016), adulteration (Ait-Kaddour, et al., 2018), bacterial contamination, fresh pork ATP and its degradable compounds (Shirai et al., 2016), aquatic product freshness (Masry et al., 2015; 2016) and chicken bacterial contamination (Sahar et al., 2011). These studies have been shown to be better than traditional fluorescence spectroscopy technology.

At present, the fluorescence spectrometer is limited by the structure and can not be used to obtain the synchronous fluorescence signal of intact eggs directly. there are few reports on nondestructive detection of egg freshness via SFS technology. Thus, the development of detection methods based on fluorescent signals related to eggs freshness is practical. In this study, the potential of SFS techniques was exploited to predict the freshness of intact eggs. The specific objectives of this study are to use a laboratorial SFS device to collect synchronous fluorescence spectra from intact eggs and to predict egg freshness.

\section{Materials and Methods}

\section{Egg samples}

In this study, fresh eggs were purchased from a local chicken farm for investigation. The eggs were divided into 5 groups and stored at $25^{\circ} \mathrm{C}$ for $1,4,7,10$ and 15 days to obtain different levels of freshness of the egg samples. At the end of storage, synchronous fluorescence spectra of the samples were collected.

\section{Collection of Synchronous Fluorescence Spectra}

In this study, Synchronous fluorescence spectra were acquired using a fluorescence spectrometer F98 (Shanghai Lengguang Technology Co., Ltd, China) supported by a laboratory SFS acquisition device (Fig. 1). Via this device, the intact egg sample's synchronous fluorescence spectrum could be measured directly. The process of measuring the fluorescence spectrum of eggs is shown in Fig. 1, the intact egg sample was positioned in a ring cage made of rubber. The excited light emitted by the xenon lamp source of the fluorescence spectrometer passes through the monochromatic exciter and the continuous monochromatic light wave is projected vertically onto the surface of the eggs through a quartz window by the excited optical fiber. Then, a 15 $\mathrm{mm}$ spot was formed on the surface of the sample. The fluorescence emitted from the sample was received by a transmitting optical receiving fiber, which was arranged at angle of $45^{\circ}$ below the quartz window. During the test, the sample was covered with a light-shielding cover to avoid interference from extraneous light.

For every sample, the SFS was obtained by measuring the emission spectra from 260 to $800 \mathrm{~nm}$ at 1 $\mathrm{nm}$ intervals, with excitation at every $10 \mathrm{~nm}$ from 260 to $800 \mathrm{~nm}$. The method of measuring a samples SFS spectrum is to excite the sample with a certain wavelength, detect the emitted light in a certain wavelength range, then change the excitation wavelength and record the emission at all wavelengths and so on. Therefore, the excitation wavelength is first fixed at $260 \mathrm{~nm}$ and the emitted light is captured at intervals of $1 \mathrm{~nm}$ at all wavelengths in the range of $260-800 \mathrm{~nm}$. Next, the excitation wavelength is set to $10 \mathrm{~nm}$ larger than the previous wave and the emission is registered at all wavelengths. For both excitation and emission, the slit width was adjusted to $10 \mathrm{~nm}$ and the scanning speed was $6000 \mathrm{~nm} / \mathrm{min}$.

\section{Freshness Measurement}

After acquisition of the SFS, the egg freshness, as expressed by the Haugh Unit (HU) (standard method for measuring egg freshness), was immediately obtained using destructive method according to Li's description (Li, 2013), All samples were randomly divided into two groups. One group accounted for $3 / 4$ of the samples, which was used for model calibration and the other group accounted for $1 / 4$ of the samples, which was used for model validation. 


\section{Preprocessing of SFS Data}

\section{Determination of the Useful Fluorescence Signal} Region

A typical averaged feature fluorescence image is shown in Fig. 2. SFS technique measuring several emission spectra at different excitation wavelengths created an Excitation-
Emission Matrix (EEM). The vertical axis represents the excitation wavelengths; the horizontal axis represents the emission wavelengths. Each horizontal line taken from the image represents an emission fluorescent spectrum for a particular excitation wavelength and the point of each excitation-emission matrix is the fluorescence intensity at the point of measurement.

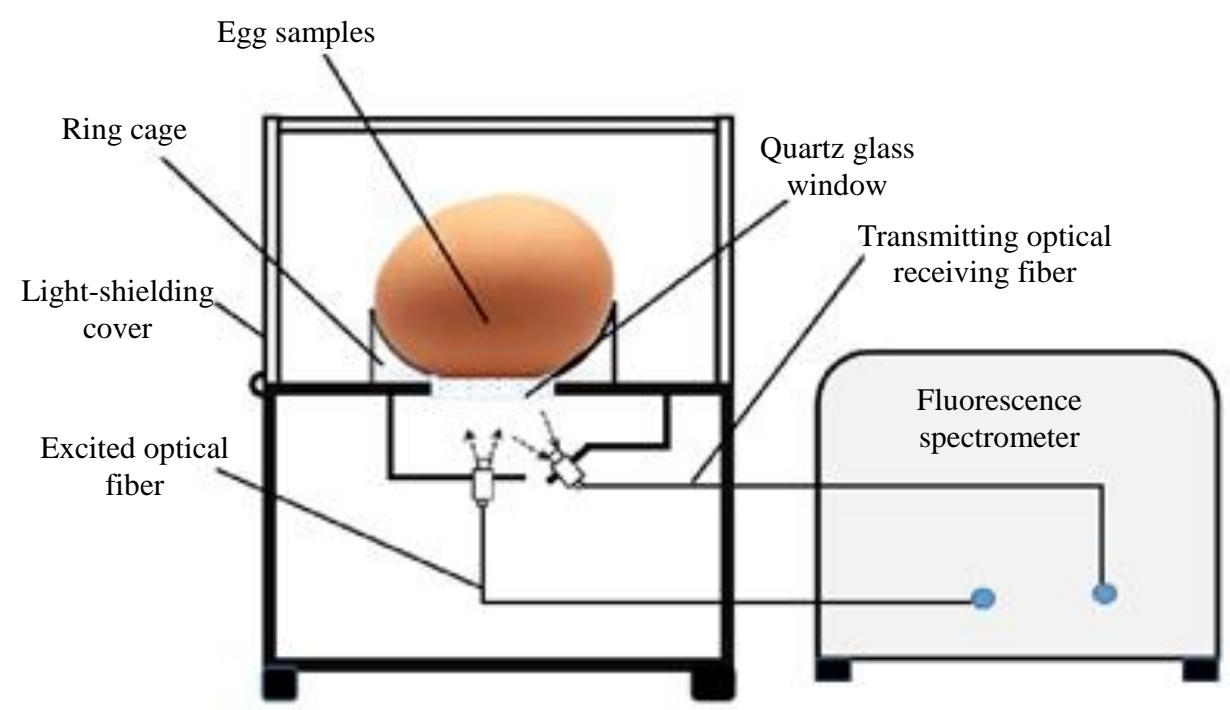

Fig. 1: Direct measurement of the fluorescence spectra of egg samples with a laboratory fluorescence acquisition device

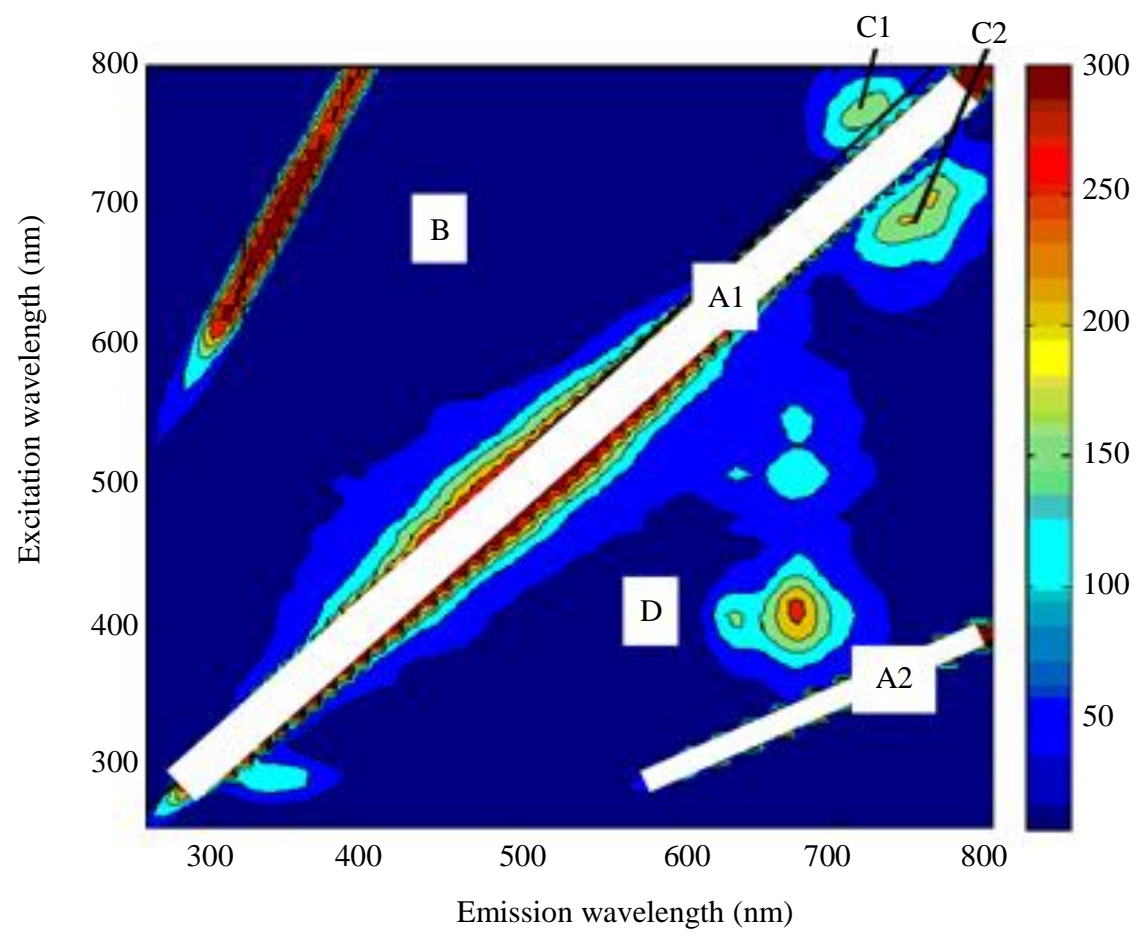

Fig. 2: Excitation-emission matrix spectrum for eggs 
In the two-dimensional SFS spectrum, some areas were caused by Raman scattering and Rayleigh scattering, which did not carry any sample information and were not useful. These areas were useless for prediction model building. First, when excited light is incident on biological tissues, Raman scattering and first and second order Rayleigh scattering is produced; these scattering signals do not contain useful chemical information and do not have any effect on subsequent modeling; therefore, the areas affected by these scattering signals can be removed from the fluorescence spectrum, as shown in region $\mathrm{A} 1$ and $\mathrm{A} 2$, the first-order scattering influence region ridge center closes to the line $\lambda_{\mathrm{Ex}}=\lambda_{\mathrm{Em}}$ and the second-order scattering influence region ridge center closes to the line $\left(\lambda_{\mathrm{Em}}=2 \lambda_{\mathrm{Ex}}\right)$, respectively. Second, according to the Stokes shift, fluorescence does not occur in the region where the excitation wavelength is greater than the emission wavelength. As shown in region B in Fig. 2, the upper left corner region, where the excitation wavelength is greater than the emission wavelength, is useless as well. In addition, on both sides of the scattering line, as shown in region $\mathrm{C} 1$ and $\mathrm{C} 2$ in Fig. 2, two strong fluorescent signal regions are symmetrically distributed due to the stray light caused by the light source. Therefore, the useful fluorescence signal in this research is in region $\mathrm{D}$.

\section{Extraction of the Ex-Em Spectrum from ROI}

Furthermore, the ROI chosen from the fluorescence image formed a 2D matrix ( $i$ excitations $\times j$ emissions). All samples were arranged to produce threedimensional data $(K \times i \times j, \mathrm{~K}$ is sample number), which was difficult for analyze further using stoichiometry analyses; therefore, it was necessary to reduce the dimension. Each excitation-emission wavelength combination was treated as one variable and Ex-Em wavelength combinations were arranged in one dimension, yielding a data array of only one dimension $X(i \times j)$ in which any value $X_{i j}$ represents the fluorescence intensity in a sample measured at emission wavelength $j$ at excitation wavelength $i$. Thus, the original 2D matrix data array for the ROI was unfolded into a one-dimensional array.

\section{Prediction Models for Egg Freshness}

Stepwise discrimination analysis is one of the most commonly used variable selection methods in multiple regression analysis. The basic concept of stepwise regression analysis is as follows: First, select some initial variable sets $(X i, 1 \leq i \leq m, m$ is the initial number of independent variables for multiple regression) from the initial variables; a variable that has the greatest influence on the dependent variable (Y) is introduced into the initial set from the other independent variables. Then, all variables that selected for multiple regression are used one by one for a significance test ( $F$ test). When the original introduced variable becomes less significant due to the introduction of a latter variable, it is eliminated. Introducing a variable or eliminating a variable is a step of stepwise discrimination and a significance test is performed at each step to ensure that every time a new variable is introduced, it is significant; only the variables that affect the significance of the dependent variable are included in the regression set. This process is repeated until no significant variable is introduced into the regression set and no significant variable is removed from the regression set (He and Liu, 2001).

In this study, the stepwise discrimination method was performed on the Ex-Em spectrum to determine the discrete Ex-Em wavelength combination. The stepwise discrimination method is a standard procedure for variable selection that uses both backward elimination and forward addition to find pertinent variables. In this method, the number of predictors retained in the final model was determined by the levels of significance assumed for the inclusion and exclusion of predictors from the model. Stepwise discrimination was performed between Ex-Em fluorescent signals and used to analyze egg freshness (HU) to choose optimal ExEm wavelength combinations. This test was conducted by Matlab 2014a in-house statistical software using a level of significance value of 0.05 for including variables, while variables were excluded from the model using a level of significance value of 0.10 . After Stepwise discrimination analysis processing, a Multiple Linear Regression (MLR) prediction model was built using florescence signals for the optimal Ex-Em wavelength combinations:

$F=f_{0}+f_{1} a_{1}+f_{2} a_{2}+\ldots+f_{i} a_{i}$

where, $F$ is the egg $\mathrm{HU}$ value to be predicted; $i=1,2$, $3, \ldots . . n ; n$ is the total number of optimal Ex-Em wavelengths; $a_{1}, a_{2}, \ldots . a_{i}$ are fluorescence intensities at the Ex-Em wavelengths; and $f_{0}, f_{1}, f_{2}, \ldots f_{i}$ are regression coefficients.

\section{Results and Analyses}

\section{Assessment of Egg Freshness}

Table 1 shows the variance in the measured $\mathrm{HU}$ for the egg samples. In this work, the calibration group contained 54 samples and the validation group contained 17 samples. 
Table: 1 Measured Haugh units of the egg samples

\begin{tabular}{lllll}
\hline group & number & Range & mean & SD \\
\hline Calibration group & 54 & $34.89-101.69$ & 66.34 & 14.93 \\
Validation group & 17 & $42.32-100.57$ & 65.88 & 15.36 \\
\hline
\end{tabular}

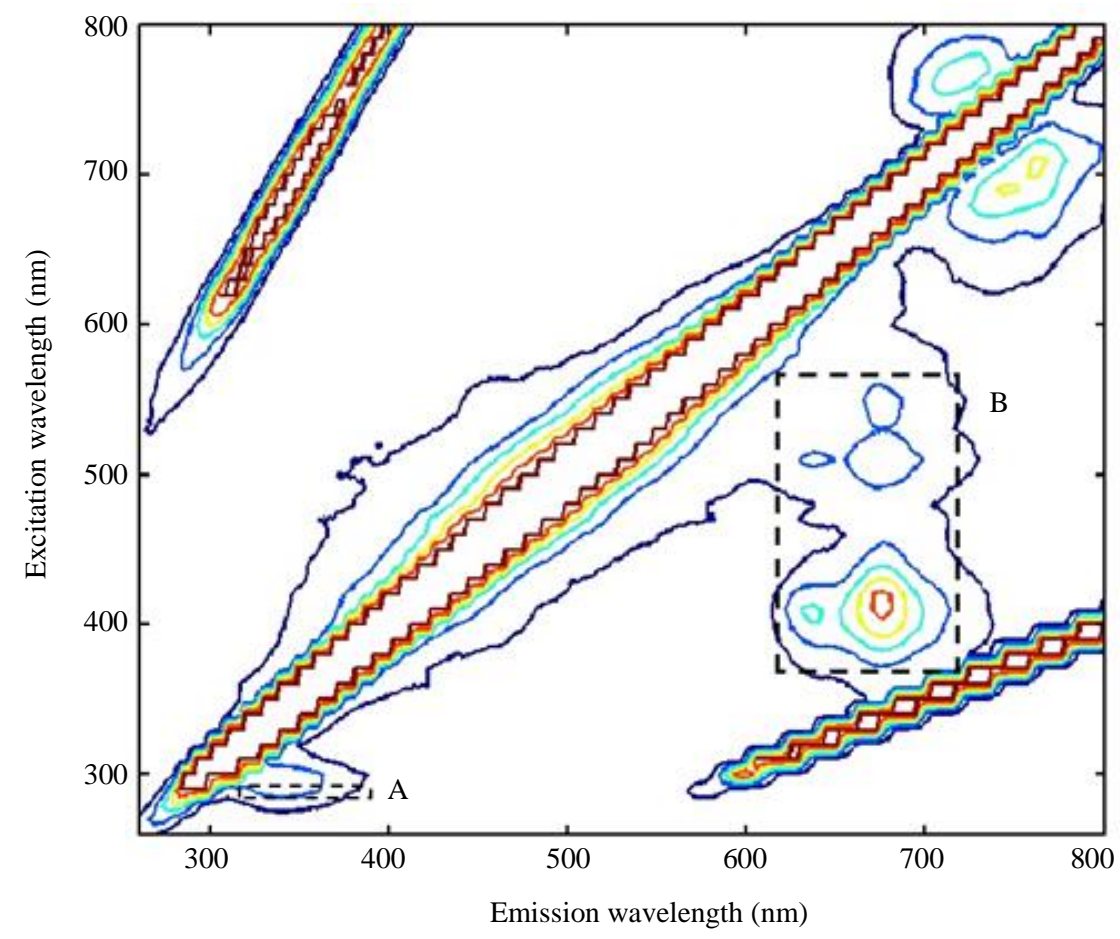

Fig. 3: Contour plot of the fluorescence intensities of eggs

\section{Determination of Regions of Interest (ROI)}

Figure 3 shows the contour plot for the fluorescence intensities; the fluorescence fingerprints of the egg samples examined in this study were characterized using various fluorescence peaks, whose intensities basically depended on the status of the samples. Through observation, we found that there were some prominent peaks, which were seen in the excitation wavelength of $290 \mathrm{~nm}$ over the emission wavelength range of 320-380 $\mathrm{nm}$ and also in the excitation wavelength range of 380 $570 \mathrm{~nm}$ over the emission wavelength range of 610-735 $\mathrm{nm}$. Especially, the contour plot for the fluorescence intensities explicitly demonstrated that all of the examined samples exhibited three different maxima for very high intensities centered at $\lambda_{\mathrm{Ex}}: 290 \mathrm{~nm} / \lambda_{\mathrm{Em}}: 345$ $\mathrm{nm}, \lambda_{\mathrm{Ex}}: 390 \mathrm{~nm} / \lambda_{\mathrm{Em}}: 675 \mathrm{~nm}$ and $\lambda_{\text {Ex }}: 490 \mathrm{~nm} / \lambda_{\mathrm{Em}}$ : $675 \mathrm{~nm}$. The intensity and shape of these fluorescence spectra were primarily dependent on the concentration of the major fluorophores in eggs, such as amino acids, proteins and pigments. Some other less intense peaks can be detected with different intensities, which varied with the freshness status of the samples, as highlighted in Fig 3. Both kinds of peaks permitted the determination of the approximate contents of the main fluorophores that were influenced during storage and affected by changes in freshness conditions.

As mentioned above, eggs had fluorescence signals that mainly concentrated in two regions: A (excitation wavelength of $290 \mathrm{~nm}$ over the emission wavelength range of $320-380 \mathrm{~nm}$ ) and $B$ (excitation wavelength range of $380-570 \mathrm{~nm}$ over the emission wavelength range of $610-735 \mathrm{~nm}$.). Egg is a complex organism, which contains a variety of fluorescence groups and its SFS signals were the comprehensive embodiment of a variety of group fluorescence signals related to its quality. Freshness is a comprehensive index of egg quality, Therefore, the two regions were selected as Regions Of Interest (ROI).

\section{Extraction of the Ex-Em Spectrum from the ROI}

Figure 4a shows the landscape of the ROI of B in the excitation wavelength range from $380-570 \mathrm{~nm}$ with an interval of $10 \mathrm{~nm}$, which had 20 wavelengths and in the emission wavelength range of $610-735 \mathrm{~nm}$ with an interval of $1 \mathrm{~nm}$, which had 126 wavelengths. The ROI for B yielded 2520 excitation-emission (ExEm) wavelength combinations (20 excitations $\times 126$ 
emissions) of which the fluorescence intensities were registered. All samples were arranged to produce three-dimensional data $(K \times i \times j, \mathrm{~K}$ is sample number), which was difficult for performing further analysis using stoichiometry, so it was necessary to reduce the dimension. Typically, the ROI for B of every sample is a $2 \mathrm{D}$ matrix, with each excitationemission wavelength combination treated as one variable and Ex-Em wavelength combinations arranged as one dimension, yielding a data array of only one dimension $X(i \times j)$ (i.e., 2520), in which any value $X_{i j}$ represents the fluorescence intensity in a sample measured at emission wavelength $j$ at excitation wavelength $\mathrm{i}$. Thus, the original $2 \mathrm{D}$ matrix data array was unfolded into a one dimensional array, as shown in Fig. 4b.

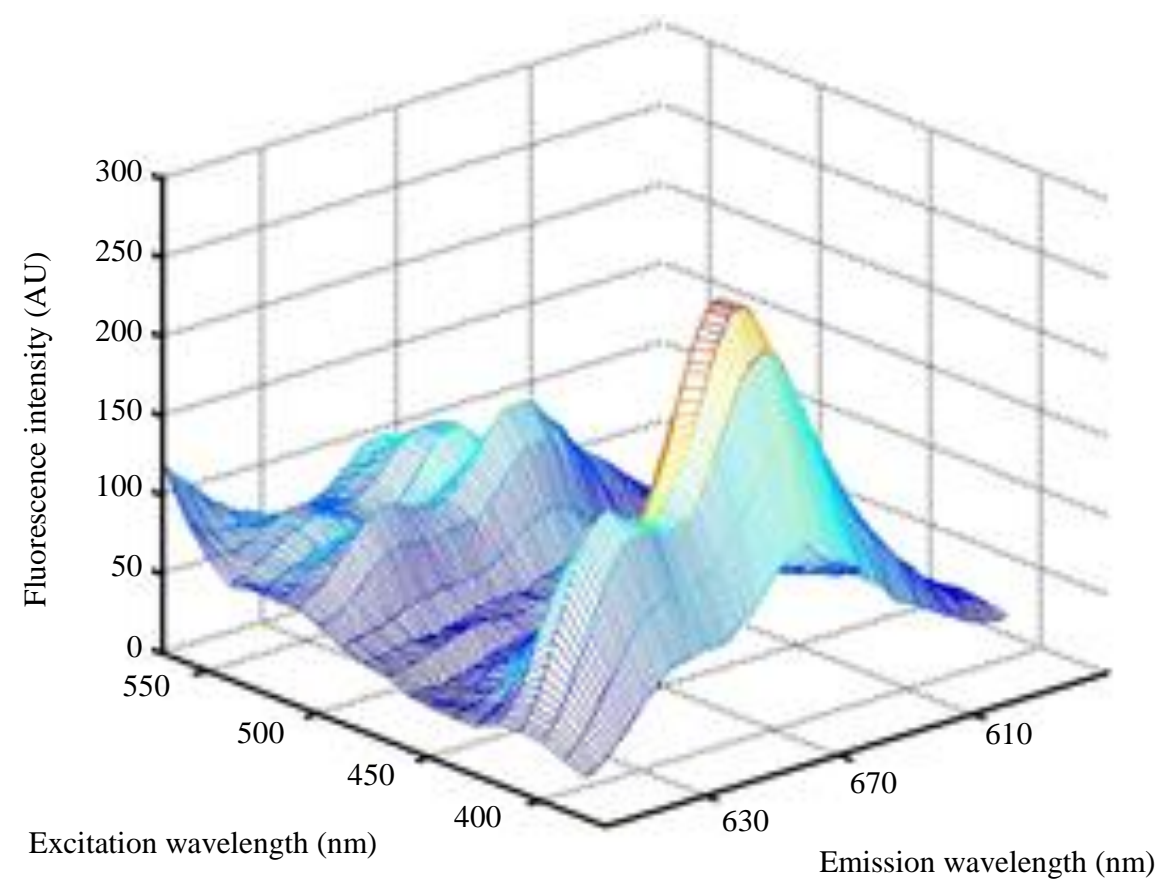

(a)

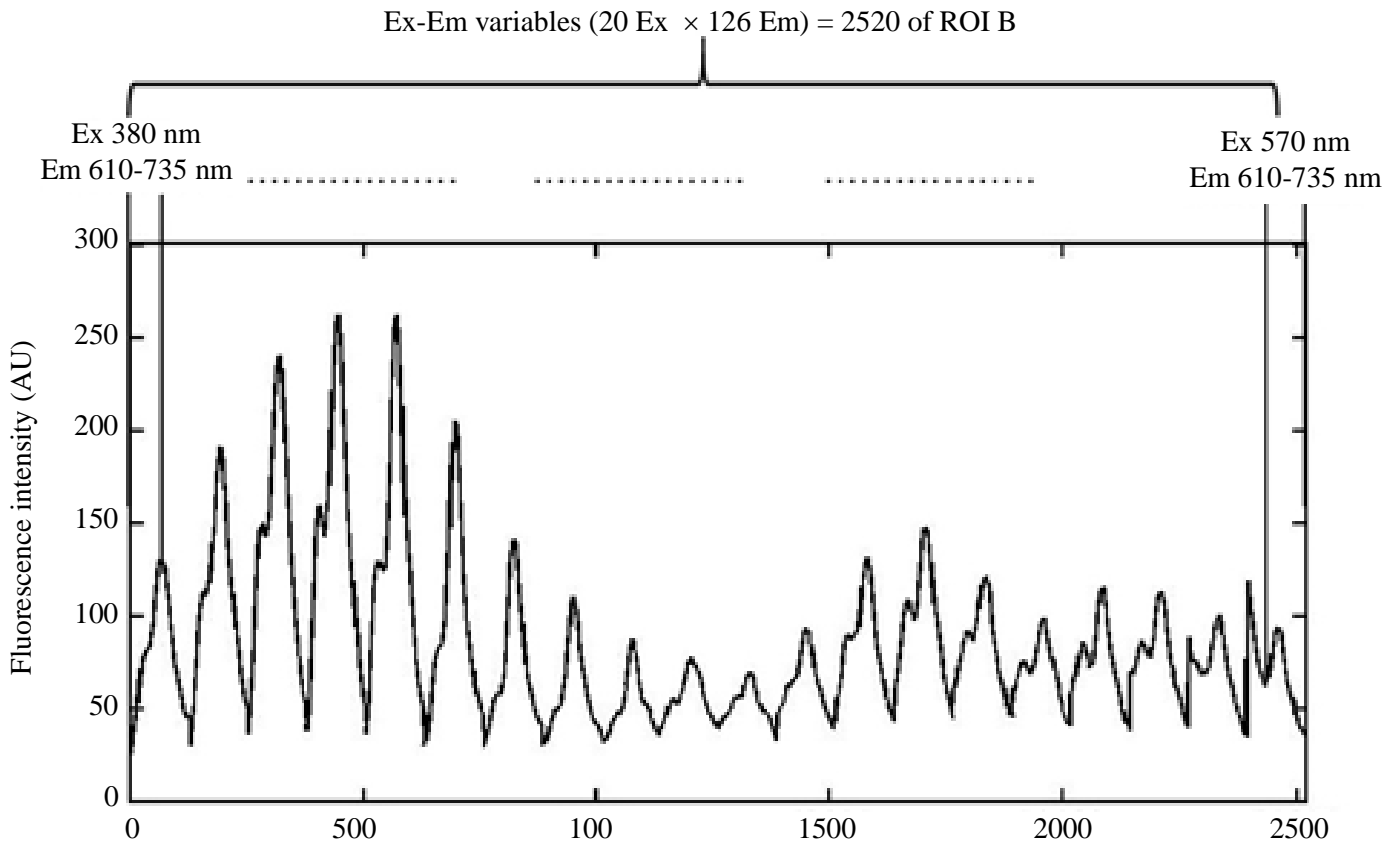

(b) 


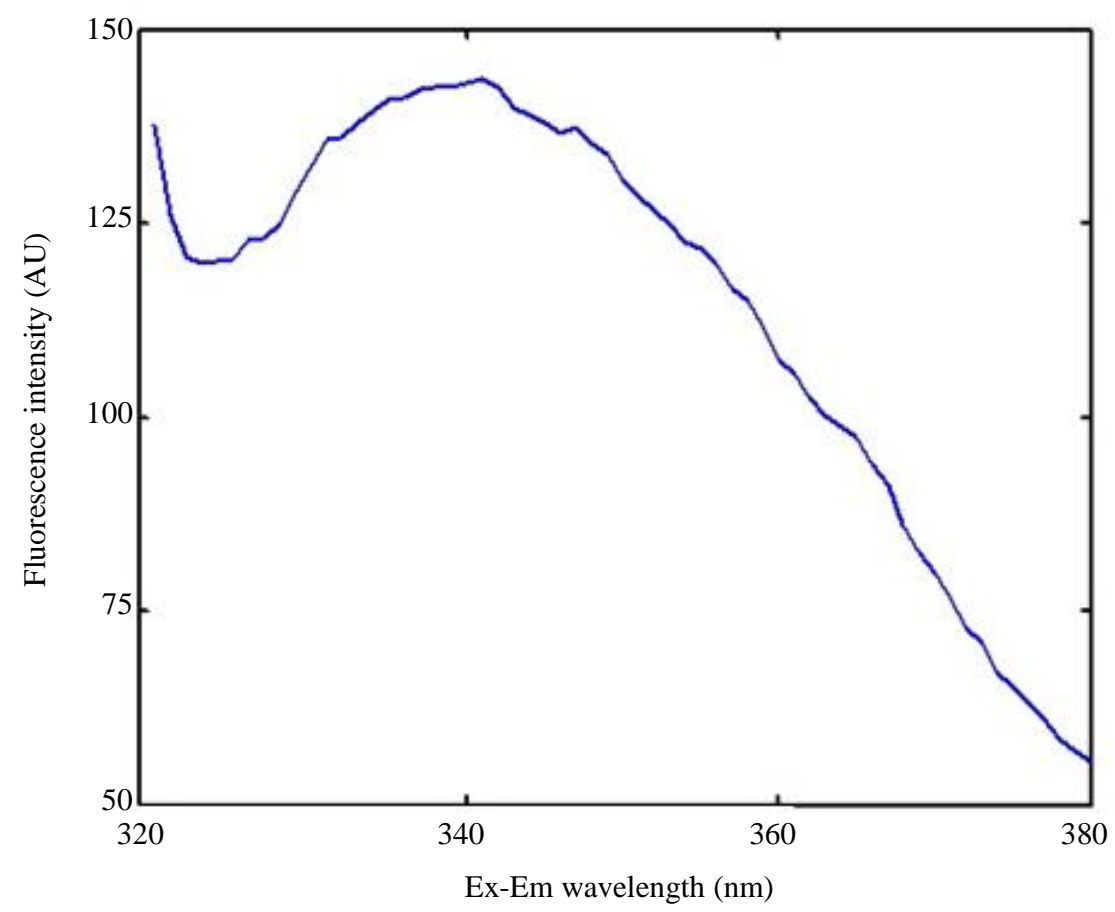

(c)

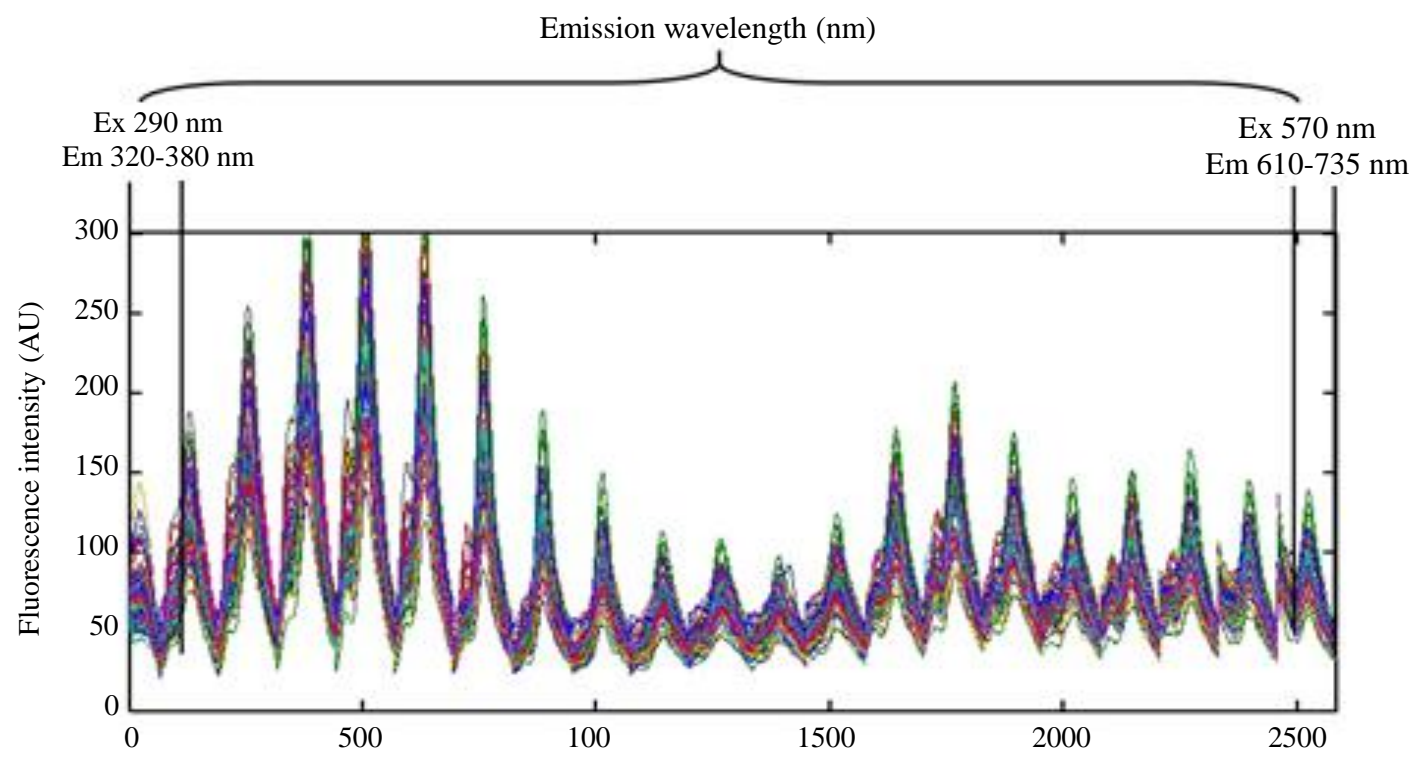

(d)

Fig. 4: Extraction of the Ex-Em spectrum; (a) The landscape of the ROI B; (b) One dimension Ex-Em fluorescence spectrum unfolded from ROI B; (c) One dimension Ex-Em fluorescence spectrum of ROI A; (d) One dimension Ex-Em fluorescence spectrum unfolded from ROI A and B of all samples

Figure $4 \mathrm{c}$ shows the excitation wavelength from the ROI of A, which was an emission spectrum with an interval of $1 \mathrm{~nm}$ that contained 61 wavelengths from 320 to $380 \mathrm{~nm}$ at an emission wavelength of $290 \mathrm{~nm}$.

The one-dimensional array unfolded from ROI A (61 Ex-Em wavelengths combinations) and ROI B A (2520
Ex-Em wavelengths combinations) were arranged in order. Finally, a one-dimensional array, which included 2581 excitation-emission wavelengths, was extracted from the fluorescence spectra of the sample. Figure $4 \mathrm{~d}$ shown the one dimension Ex-Em fluorescence spectrum unfolded from ROI A and B of all samples. 


\section{Simple Correlation Analysis between Eggs Freshness and Ex-Em Spectrum}

Before using the stepwise regression discriminant method to select the characteristic Ex-Em wavelength combination for MLR prediction model construction, the simple correlation analysis between the fluorescence signal at each excitation-emission wavelength and the freshness of eggs was analyzed. The results are shown in Fig. 5. It can be seen from the Fig. 5 that there is a great difference between Ex-Em fluorescence signal and egg freshness in the whole Ex-Em spectrum range. According to statistics, there are 1329 Ex-Em fluorescence signal points where the absolute value of correlation coefficient ( $R$ value) is greater than 0.6 and there are 313 Ex-Em fluorescence signal points where the $\mathrm{R}$ values is greater than 0.6 , furthermore, the $\mathrm{R}$ values at $33 \mathrm{Ex}$-Em fluorescence signal points are more than 0.70 . The Ex-Em spectra with $\mathrm{R}$ values greater than 0.70 are mainly concentrated at the emission wavelengths of $380 \mathrm{~nm}, 450 \mathrm{~nm}, 460 \mathrm{~nm}$ and $490 \mathrm{~nm}$. The maximum $R$ values of the Em spectra of the $4 \mathrm{Ex}$ wavelengths appear at at $735,650,725$ and $730 \mathrm{~nm}$ are $0.734,0.7185,0.7356$ and 0.7466 respectively. This showed a good correlation between the Ex-Em fluorescence spectrum signal extracted from the selected ROI region and the freshness of eggs.

\section{Prediction of Egg Freshness (Haugh Unit)}

The stepwise method was performed to determine the optimal Ex-Em wavelengths. Discrete Ex-Em wavelength combinations were identified as sensitive variables for predicting the Haugh units for an egg. The combinations, which included 27 emission wavelengths, were excited using 13 different excitation wavelengths (Table 2).

Table: 2 Determination of Ex-Em wavelengths combinations

\begin{tabular}{lll}
\hline & Excitation wavelength $(\mathrm{nm})$ & Emission wavelength(nm) \\
\hline 1 & 290 & 320,335 \\
2 & 380 & 731,735 \\
3 & 400 & $643,721,728$ \\
4 & 410 & $628,633,643,676,735$ \\
5 & 430 & 667 \\
6 & 450 & 667,727 \\
7 & 460 & $622,662,693,699,724,731$ \\
8 & 470 & 702 \\
9 & 490 & 667 \\
10 & 500 & 743 \\
11 & 510 & 745 \\
12 & 520 & 635 \\
13 & 530 & 635 \\
\hline
\end{tabular}

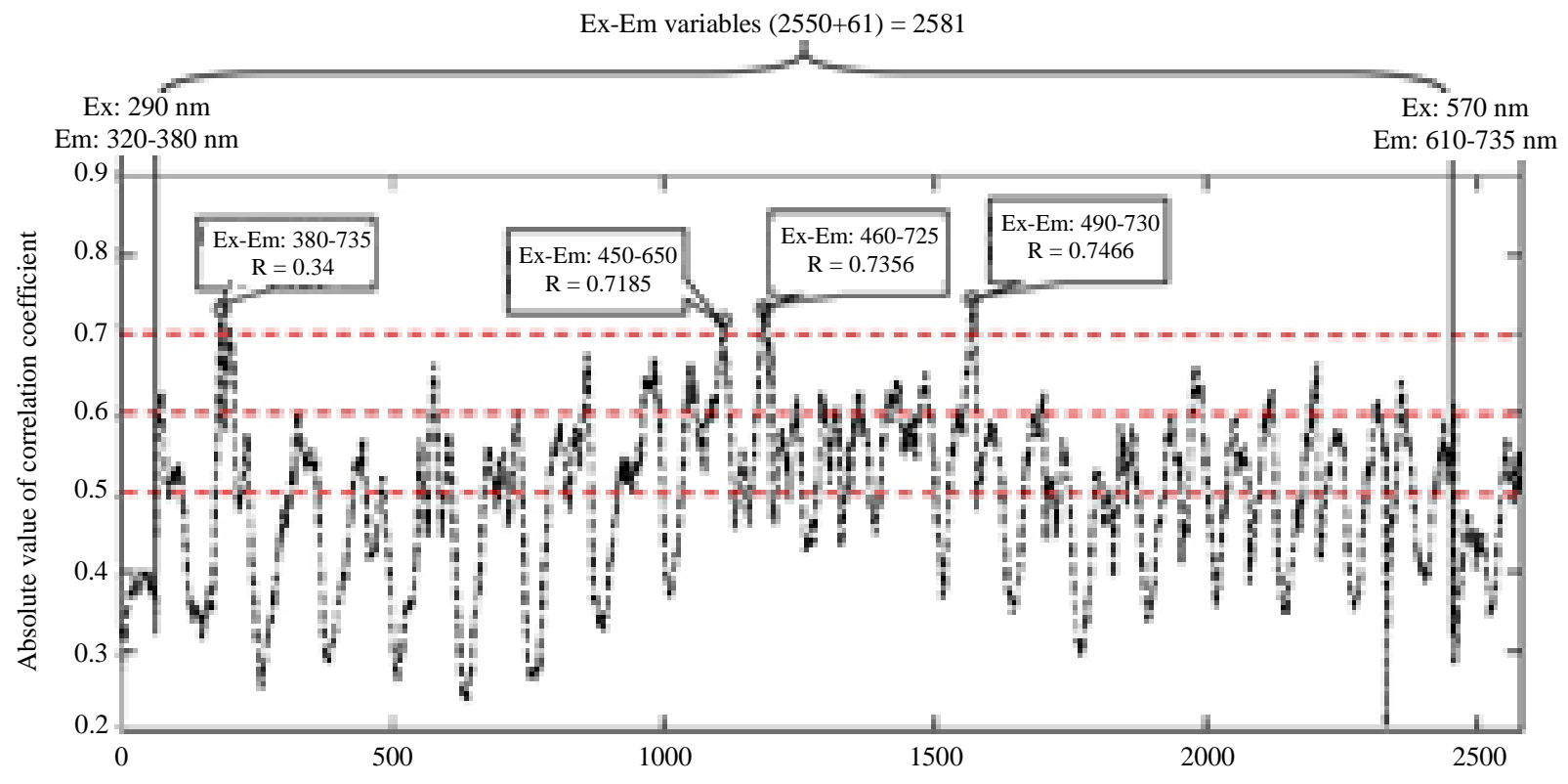

Fig. 5: Sample correlation analyze curve between eggs freshness value and Ex-Em spectrum 


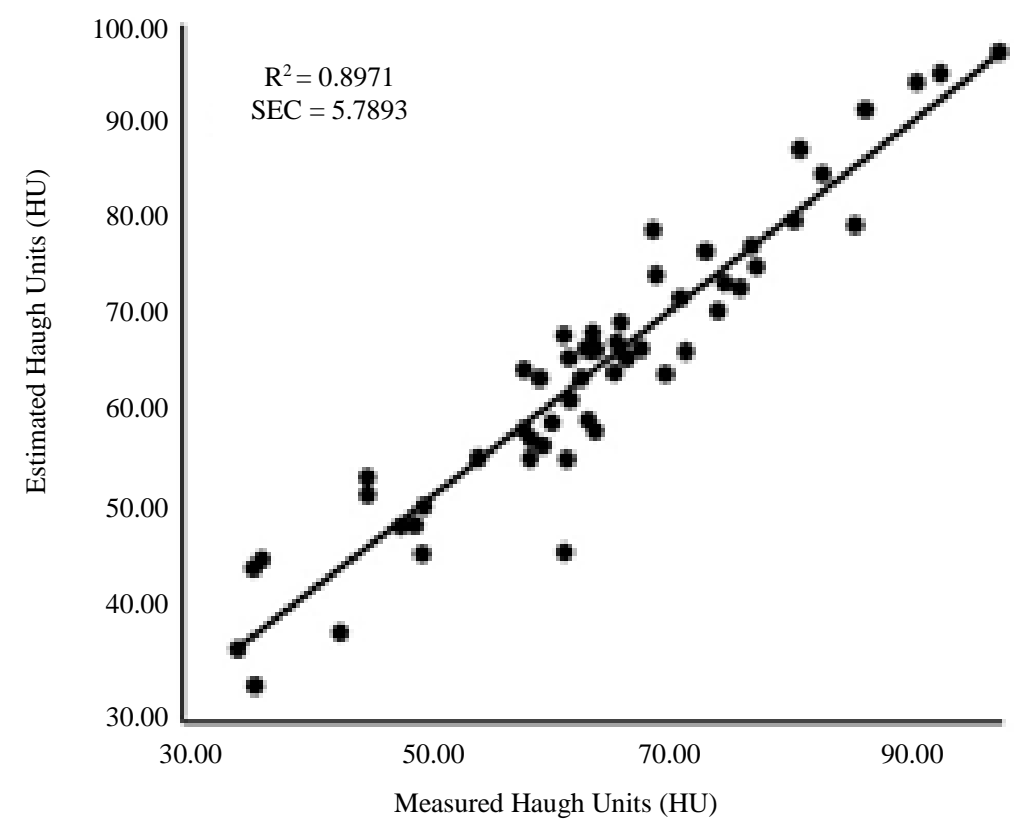

(a)

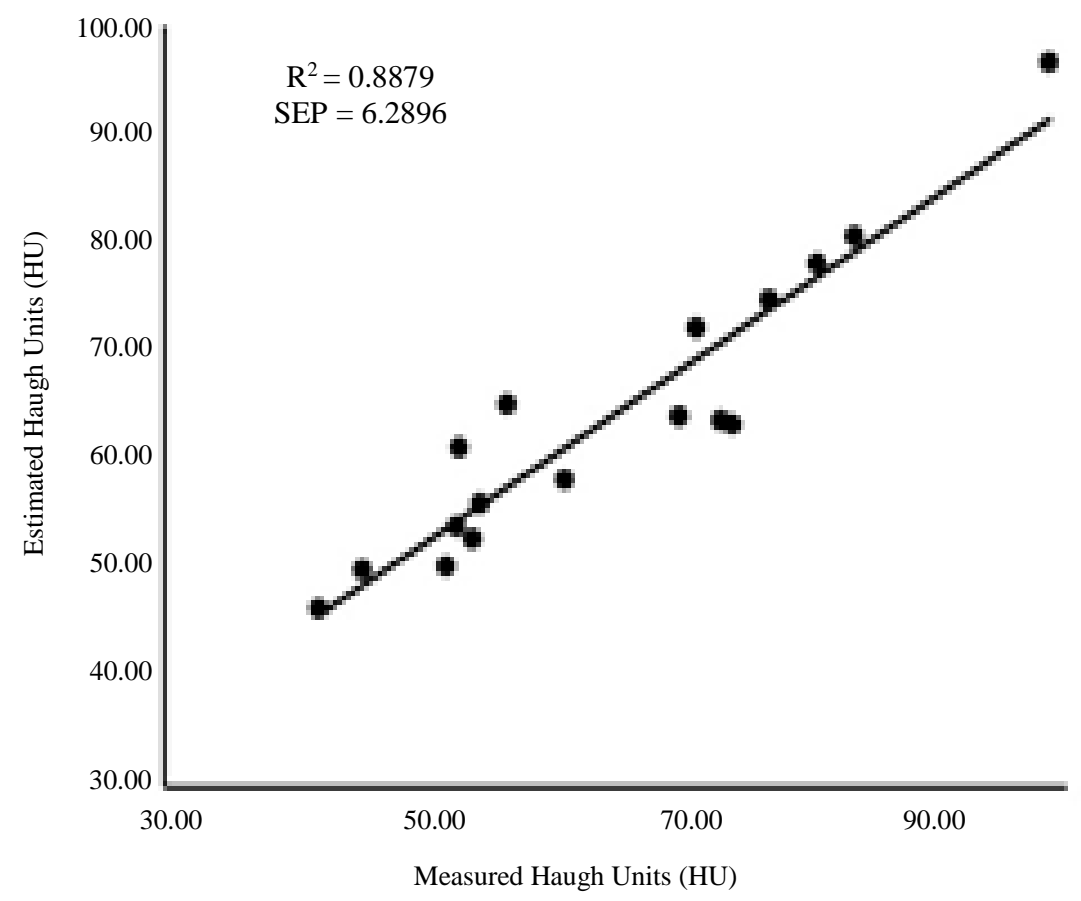

(b)

Fig. 6: Egg freshness calibration and validation results for MLR

MLR prediction models were established based on the Ex-Em wavelength combinations of the optimal wavelengths, which consisted of a constant term and regression coefficients corresponding to the optimal wavelength numbers. Then, validation was performed to validate the predictive accuracy of the models.
Figure 6 shows the egg freshness calibration and validation results for MLR based on the Ex-Em wavelength combinations of 27 optimal wavelengths.

The MLR model gave good predictions for the calibration, with $\mathrm{R}^{2}=0.8971$ and $\mathrm{SEC}=5.7893$ (Fig. 6a). The model predicted egg freshness with $R_{p}{ }^{2}=0.91$ 
and SEP = 6.2896 (Fig. 6b). The results of Haugh units and synchronous fluorescence spectral measurement were highly correlated in MLR model.

\section{Conclusion}

In this study, a laboratory $S F S$ acquisition device is developed and SFS of intact eggs were collected over the wavelength range from 260 to $800 \mathrm{~nm}$. Eggs had fluorescence signals that were mainly concentrated in two regions: A (excitation wavelength of $290 \mathrm{~nm}$ over the emission wavelength range of 320-380 nm) and B (excitation wavelength range of $380-570 \mathrm{~nm}$ over the emission wavelength range of $610-735 \mathrm{~nm}$.); the two regions were selected as Regions Of Interest (ROI) with a one dimension array, which included 2581 excitationemission wavelengths, extracted from the sample ROI fluorescence spectra. Stepwise discrimination analysis was performed and 27 Ex-Em wavelength combinations were determined. A Multiple Linear Regression (MLR) prediction model was built using fluorescence signals based on the 27 optimal Ex-Em wavelength combinations. The results revealed that the freshness of an egg could be accurately predicted with $\mathrm{Rp}^{2}$ of 0.8879 and a root mean square error estimated by validation (SEP) of 6.2896. The model prediction results can meet the requirements of practical detection and application. This study demonstrates that the synchronous fluorescence spectral technique has high potential for nondestructive sensing of egg freshness.

At present, the fluorescence spectrometer is limited by the structure and can not be used to obtain the SFS signal of intact eggs directly. The SFS acquisition device and analysis method developed in this study demonstrates a methodology for the future development Synchronous fluorescence nondestructive detection. This is an sensitive egg freshness detection method. Egg is a complex organism, which contains a variety of fluorescence groups; The change of synchronous fluorescence spectral was affected by the egg's age, variety, feeding conditions and so on. In the future research, it is necessary to consider the variety of egg samples, breeding age and the feeding conditions, in order to meet the needs of practical detection applications.

\section{Acknowledgment}

The authors gratefully acknowledge the "National Key Research and Development Program of China" (Project No. 2017YFC1600801) for supporting this research.

\section{Author's Contributions}

Jianhu Wu and Guifeng Li: Designed and developed the method, performed the numerical experiments, analyzed the data and wrote the paper.
Yankun Peng, Junjie Du: Performed the numerical experiments and revised the manuscript.

Jianguo Xu and Gang Gao: prepared the experimental equipment and revised the manuscript.

\section{Ethics}

The authors declare their responsibility for any ethical issues that may arise after the publication of this manuscript.

\section{Conflict of Interest}

The authors declare that they have no competing interests. The corresponding author affirms that all of the authors have read and approved the manuscript.

\section{References}

Aït-Kaddou, A., T. Boubellouta and I. Chevallier, 2011. Development of a portable spectrofluorimeter for measuring the microbial spoilage of minced beef. Meat Sci., 88: 675-681.

DOI: 10.1016/j.meatsci.2011.02.027

Aït-Kaddour, A., A. Thomas, J. Mardon, D. Jacquot and A. Ferlay et al., 2016. Potential of fluorescence spectroscopy to predict fatty acid composition of beef. Meat Sci., 113: 124-131.

DOI: $10.1016 /$ j.meatsci.2015.11.020

Aït-Kaddour, A., M. Loudiyi, A. Ferlay and D. Gruffat, 2018. Performance of fluorescence spectroscopy for beef meat authentication: Effect of excitation mode and discriminant algorithms. Meat Sci., 137: 58-66. DOI: 10.1016/j.meatsci.2017.11.002

Asylbek, K., D. Bertrand, J. Lepetit, A. Listrat and A. Laguet et al., 2012. Potential of a custom-designed fluorescence imager combined with multivariate statistics for the study of chemical and mechanical characteristics of beef meat. Food Chem., 131: 1030-1036. DOI: 10.1016/j.foodchem.2011.09.066

Bamelis, F.R., B.J. Kemps and K. Mertens, 2003. Non destructive internal egg freshness assessment using VIS-NIR spectroscopy. Proceedings of 10th European Symposium on the Quality of Eggs and Egg Products, Saint-Brieuc Ploufragan, France, pp: 91-97.

Bjørg, E., P.B. Wold and A. Sponnich, 2002. On attempts to measure the tenderness of LongissimusDorsi muscles using fluorescence emission spectra. Meat Sci., 60: 187-202.

DOI: $10.1016 /$ S0309-1740(01)00121-8

Brøndum, J., L. Munck, P. Henckel, A. Karlsson and E. Tornberg et al., 2000. Prediction of water-holding capacity and composition of porcine meat by comparative spectroscopy. Meat Sci., 55: 177-185. DOI: 10.1016/S0309-1740(99)00141-2 
Dufour, É., J.P. Frencia and E. Kane, 2003. Development of a rapid method based on front-face fluorescence spectroscopy for the monitoring of fish freshness. Food Res. Int., 36: 415-423. DOI: 10.1016/S0963-9969(02)00174-6

Durek, J., A. Fröhling, J. Bolling, R. Thomasius and P. Durek et al., 2016. Non-destructive mobile monitoring of microbial contaminations on meat surfaces using porphyrin fluorescence intensities. Meat Sci., 115: 1-8. DOI: 10.1016/j.meatsci.2015.12.022

Durek, J., J.S. Bolling, D. Knorr, F. Schwägele and O. Schlüter, 2012. Effects of different storage conditions on quality related porphyrin fluorescence signatures of pork slices. Meat Sci., 90: 252-258.

DOI: 10.1016/j.meatsci.2011.07.010

Gatellier, P., S. Gomez, V. Gigaud, C. Berri and E.L. Bihan-Duval et al., 2007. Use of a fluorescence front face technique for measurement of lipid oxidation during refrigerated storage of chicken meat. Meat Sci., 76: 543-547. DOI: 10.1016/j.meatsci.2007.01.006

Gatellier, P., V. Santé-Lhoutellier, S. Portanguen and A. Kondjoyan, 2009. Use of meat fluorescence emission as a marker of oxidation promoted by cooking. Meat Sci., 83: 651-656. DOI: 10.1016/j.meatsci.2009.07.015

He, X.Q. and W.Q. Liu, 2001. Applied regression analysis. China Renmin University Press, Beijing.

Kemps, B.J., F.R. Bamelis and B.D. Ketelaere, 2006. Visible transmission spectroscopy for the assessment of egg freshness. J. Sci. Food Agric., 86: 1399-1406. DOI: $10.1002 /$ jsfa.2528

Kulmyrzaev, A., D. Bertrand, J. Lepetit, A. Listrat and A. Laguet et al., 2012. Potential of a custom-designed fluorescence imager combined with multivariate statistics for the study of chemical and mechanical characteristics of beef meat. Food Chem., 131: 1030-1036. DOI: 10.1016/j.foodchem.2011.09.066

Lenhardt, L., R. Bro, I. Zeković, T. Dramićanin and T. Dramićanin et al., 2015. Fluorescence spectroscopy coupled with PARAFAC and PLS DA for characterization and classification of honey. Food Chem., 175: 284-291.

DOI: 10.1016/j.foodchem.2014.11.162

Li, B.N., H. Wang, Q. Zhao, J. Ouyang and Y. Wu, 2015. Rapid detection of authenticity and adulteration of walnut oil by FTIR and fluorescence spectroscopy: A comparative study. Food Chem., 181: 25-30.

DOI: 10.1016/j.foodchem.2015.02.079

Li, X.L., 2013. The Haugh Unit is an important indicator of the egg quality inspection. J. Inspect. Quarantine, 23: 48-50.

Mala, D.M., M. Yoshimura, S. Kawasaki, M. Tsuta and M. Kokawa et al., 2016. Fiber optics fluorescence fingerprint measurement for aerobic plate count prediction on sliced beef surface. LWT-Food Sci. Technol., 68: 14-20. DOI: 10.1016/j.lwt.2015.11.065
Masry, G.E., H. Nagai, K. Moria, N. Nakazawa and M. Tsuta et al., 2015. Freshness estimation of intact frozen fish using fluorescence spectroscopy and chemometrics of excitation-emission matrix. Talanta, 143: 145-156. DOI: 10.1016/j.talanta.2015.05.031

Masry, G.E., N. Nakazaw, E. Okazaki and S. Nakauchi, 2016. Non-invasive sensing of freshness indices of frozen fish and fillets using pretreated excitationemission matrices. Sensors Actuators B: Chemical, 228: 237-250. DOI: 10.1016/j.snb.2016.01.032

Oto, N., S. Oshita, Y. Makino, J. Sugiyama and M. Yoshimura, 2013. Non-destructive evaluation of ATP content and plate count on pork meat surface by fluorescence spectroscopy. Meat Sci., 93: 579-585. DOI: 10.1016/j.meatsci.2012.11.010

Pouzo, L.B., N.E. Zaritzky, E. Pavan, L. Rossetti and A.M. Descalzo, 2016. Utilization of fluorescence spectroscopy as a novel approach to evaluate the oxidative stability in beef retail displayed. Meat Sci., 119: 7-13. DOI: 10.1016/j.meatsci.2016.03.027

Sahar, A. and É. Dufour, 2015. Classification and characterization of beef muscles using front-face fluorescence spectroscopy. Meat Sci., 100: 69-72. DOI: 10.1016/j.meatsci.2014.09.142

Sahar, A., T. Boubellouta, J. Lepetit and É. Dufour, 2009. Front-face fluorescence spectroscopy as a tool to classify seven bovine muscles according to their chemical and rheological characteristics. Meat Sci., 83: 672-677. DOI: 10.1016/j.meatsci.2009.08.002

Sahar, A., T.É. Boubellouta and É. Dufour, 2011. Synchronous front-face fluorescence spectroscopy as a promising tool for the rapid determination of spoilage bacteria on chicken breast fillet. Food Res. Int., 44: 471-480. DOI: 10.1016/j.foodres.2010.09.006

Sahar, A., U. Rahman, A. Kondjoyan, S. Portanguen and É. Dufour, 2016. Monitoring of thermal changes in meat by synchronous fluorescence Spectroscopy. J. Food Eng., 168: 160-165. DOI: 10.1016/j.jfoodeng.2015.07.038

Schneider, J., J. Wulf, B. Surowsky, H. Schmidt and F. Schwägele et al., 2005. Fluorimetric detection of protoporphyrins as an indicator for quality monitoring of fresh intact pork meat. Meat Sci., 69: 35-46.

Shirai, H., S.S. Oshita and Y. Makino, 2016. Detection of fluorescence signals from ATP in the second derivative excitation-emission matrix of a pork meat surface for cleanliness evaluation. J. Food Eng., 168: 173-179. DOI: 10.1016/j.jfoodeng.2015.07.032

Zhao, J., H. Lin, Q. Chen, X. Huang and Z.B. Sun et al., 2010. Identification of egg's freshness using NIR and support vector data description. J. Food Eng., 98: 408-414. DOI: 10.1016/j.jfoodeng.2010.01.018 